\title{
Random Walks on the Affine Group of a Homogeneous Tree in the Drift-Free Case
}

\author{
Dariusz Buraczewski • Konrad Kolesko
}

Received: 16 December 2009 / Revised: 16 September 2010 / Published online: 19 October 2010

(C) The Author(s) 2010. This article is published with open access at Springerlink.com

\begin{abstract}
The affine group of a homogeneous tree is the group of all its isometries fixing an end of its boundary. We consider a random walk with law $\mu$ on this group and the associated random processes on the tree and its boundary. In the drift-free case there exists on the boundary of the tree a unique $\mu$-invariant Radon measure. In this paper we describe its behaviour at infinity.
\end{abstract}

Keywords Random walk · Affine group · Homogeneous tree · Invariant measure

Mathematics Subject Classification (2000) Primary 60B15

\section{Introduction}

Let $\mathbb{T}=\mathbb{T}_{q}$ be a homogeneous tree of degree $q+1$. We denote by $\operatorname{Aff}(\mathbb{T})$ the group of affine transformations of the tree $\mathbb{T}$, that is the group of isometries of the tree that fix an end $\omega$ of the boundary. This group is locally compact, totally disconnected, amenable and non-unimodular. The group Aff $(\mathbb{T})$ is an analogue of the real affine group acting on the hyperbolic plane $\mathbb{H}^{2}$ by isometries and fixing a boundary point. However, its structure is much more difficult. If $q$ is a prime number $p$, then the group Aff $(\mathbb{T})$ contains on one side the affine group of $p$-adic numbers $\operatorname{Aff}\left(\mathbb{Q}_{p}\right)$ (i.e. the group of matrices of the form $\left[\begin{array}{ll}a & b \\ 0 & 1\end{array}\right]$, where $a, b$ are $p$-adic numbers and $a$ is nonzero), which in some sense is similar to $\operatorname{Aff}(\mathbb{R})$, but on the other hand it contains groups

This research project has been partially supported by MNiSW grant N N201 393937.

D. Buraczewski · K. Kolesko (凶)

Instytut Matematyczny, Uniwersytet Wrocławski, pl. Grunwaldzki 2/4, 50-384 Wrocław, Poland e-mail:kolesko@math.uni.wroc.pl

D. Buraczewski

e-mail: dbura@math.uni.wroc.pl 
having completely different structure like the lamplighter group or automata groups (see [4] for further information on the structure of Aff $(\mathbb{T})$ ).

In this paper we study random walks on the affine group and related random processes on the tree $\mathbb{T}$ and its boundary $\partial \mathbb{T}$. Our goal is to describe asymptotic properties of its invariant measure. Given a probability measure $\mu$ on Aff( $\mathbb{T})$ we consider the left and the right random walk on $\operatorname{Aff}(\mathbb{T})$, i.e. sequences of random variables on the group $L_{n}=X_{n} \ldots X_{1}$ and $R_{n}=X_{1} \ldots X_{n}$, where $X_{i}$ are i.i.d. with law $\mu$. Choosing a point $o \in \mathbb{T}$ one can define random processes on the tree $L_{n} \cdot o$ and $R_{n} \cdot o$. Cartwright, Kaimanovich and Woess [4] proved that if the random process has a drift in a proper direction (all the details will be given in Sect. 2.4), then $R_{n} \cdot o$ converges almost surely to a random element of $\partial^{*} \mathbb{T}=\partial \mathbb{T} \backslash\{\omega\}$. The limit defines a harmonic probability measure, whose asymptotic properties has been recently described by Kolesko [6]. If the measure $\mu$ has a drift towards the end $\omega$ or has no drift, then $R_{n} \cdot o$ converges to $\omega$ a.s. However, to obtain more precise information about the random walk on Aff $(\mathbb{T})$ one has to consider its action on the boundary $\partial^{*} \mathbb{T}$. In the drift-free case Brofferio [1] proved, under some additional assumptions, that there exists an invariant Radon measure $v$ on $\partial^{*} \mathbb{T}$, i.e. a measure such that

$$
v(f)=\mu * v(f)=\int_{\operatorname{Aff}(\mathbb{T})} \int_{\partial^{*} \mathbb{T}} f(\gamma \mathfrak{u}) v(d \mathfrak{u}) \mu(d \gamma)
$$

for any $f \in C(\mathbb{T})$. This measure is unique (up to a multiplicative constant) and is unbounded on $\partial^{*} \mathbb{T}$. The measure $v$ in a natural way appears in the renewal theorem for the affine group, namely its small modifications are limits of the potential kernel (see [1] for more details). Therefore in the context of studying random walks on affine groups of homogeneous trees it is necessary to ask about precise description of the measure $v$.

The main goal of this paper is to study asymptotic behaviour of the measure $v$. In terms of a natural ultrametric distance on the boundary, our main result (Theorem 2.2) says that, on annuli of fixed centre and constant width, the invariant measure is asymptotically constant, and nonzero. Our proof bases partially on methods developed in $[2,3]$, where similar problems concerning the random difference equation on $\mathbb{R}^{d}$ were studied.

\section{Random Walk Walks on the Affine Group of a Tree and the Main Theorem}

\subsection{Oriented Tree}

The homogeneous tree $\mathbb{T}=\mathbb{T}_{q+1}$ of degree $q+1$ is the connected graph without any cycles whose vertices have exactly $q+1$ neighbours. For any couple of vertices $x$ and $y$ there exists exactly one sequence of successive vertices without repetition $x=x_{0}, x_{1}, \ldots, x_{k}=y$ denoted by $\overline{x y}$. Then we say that the distance between $x$ and $y$ is equal to $k$ and we write $d(x, y)=k$. A geodesic ray is an infinite sequence of successive neighbours $x_{0}, x_{1}, x_{2}, \ldots$ without repetition. Two rays are equivalent if they differ only by finitely many vertices. An end is an equivalent class of this 
relation, and the set of all ends will be denoted by $\partial \mathbb{T}$. For $\mathfrak{u} \in \partial \mathbb{T}$ and $x \in \mathbb{T}$ there exists a unique geodesic ray $\overline{x \mathfrak{u}}$ which represents $\mathfrak{u}$.

We choose and fix once for all an end $\omega$ and define $\partial^{*} \mathbb{T}=\partial \mathbb{T} \backslash\{\omega\}$. For $x, y \in$ $\mathbb{T} \cup \partial^{*} \mathbb{T}$ by $x \wedge y$ we denote the first common vertex of $\overline{x \omega}$ and $\overline{y \omega}$ i.e. $x \wedge y=z$ if $\overline{x \omega} \cap \overline{y \omega}=\overline{z \omega}$. We may imagine the oriented tree as a genealogical tree where $\omega$ is a mythical ancestor, every vertex has one ancestor and $q$ children. Let us fix a reference vertex $o$ in $\mathbb{T}$ called origin. The height function $h$ from $\mathbb{T}$ to $\mathbb{Z}$ is $h(x)=$ $d(x, x \wedge o)-d(o, x \wedge o)$, also known as Busemann function. The function $h$ induces an ultra-metric distance $\Theta$ on $\mathbb{T} \cup \partial^{*} \mathbb{T}$, for $x, y \in \mathbb{T} \cup \partial^{*} \mathbb{T}$ we define

$$
\Theta(x, y):= \begin{cases}q^{-h(x \wedge y)} & \text { if } x \neq y \\ 0 & \text { if } x=y .\end{cases}
$$

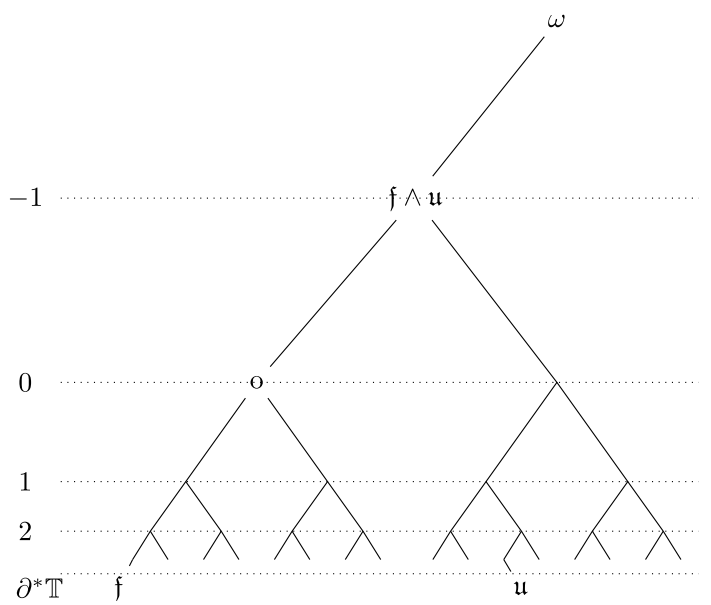

\subsection{The Affine Group}

Every isometry of $(\mathbb{T}, d)$ has a natural extension to the boundary so we can define the affine group of the tree $\mathbb{T}$ as the group of all isometries fixing the chosen end $\omega$

$$
\operatorname{Aff}(\mathbb{T}):=\{g \in \operatorname{Iso}(\mathbb{T}): g \omega=\omega\}
$$

The group Aff( $\mathbb{T})$ is equipped with the topology of pointwise convergence. To simplify our notation we will write $G$ instead of Aff $(\mathbb{T})$.

All elements of the affine group preserve the order and the distance, therefore $h(x)-h(y)=h(g x)-h(g y)$, for any couple $x, y \in \mathbb{T}$ and $g \in G$. So we may define a homomorphism $\phi$ of $G$ into $\mathbb{Z}: \phi(g)=h(g x)-h(x)=h(g o)$ and by the remark above the definition does not depend on the particular choice of $x$ and $o$. Moreover

$$
\Theta(g x, g y)=q^{-h(g x \wedge g y)}=q^{-\phi(g)} \Theta(x, y) .
$$

The horocyclic group of the tree is the subgroup of the affine group that fixes the height

$$
\operatorname{Hor}(\mathbb{T}):=\operatorname{ker} \phi=\{g \in G: h(g x)=h(x), \quad \forall x \in \mathbb{T}\}
$$


Let us fix a $\sigma \in \operatorname{Aff}(\mathbb{T})$ such that $\phi(\sigma)=1$ and $\sigma(o)$ is one of children of $o$. Every element $g \in \operatorname{Aff}(\mathbb{T})$ has a unique decomposition as a product of an element of the horocyclic group and a power of $\sigma$ :

$$
g=\left(g \sigma^{-\phi(g)}\right) \sigma^{\phi(g)} .
$$

We identify the group generated by $\sigma$ with $\mathbb{Z}$. The affine group can be decomposed into the semidirect product of $\operatorname{Hor}(\mathbb{T})$ and $\mathbb{Z}$

$$
\begin{gathered}
\operatorname{Hor}(\mathbb{T}) \rtimes \mathbb{Z} \cong \operatorname{Aff}(\mathbb{T}) \\
(\beta, m) \mapsto \beta \sigma^{m},
\end{gathered}
$$

where the action of $\mathbb{Z}$ on $\operatorname{Hor}(\mathbb{T})$ is given by $m \beta=m(\beta):=\sigma^{m} \beta \sigma^{-m}$. Then the multiplication in the affine group is given by the following formula:

$$
\left(\beta_{1}, m_{1}\right)\left(\beta_{2}, m_{2}\right)=\beta_{1} \sigma^{m_{1}} \beta_{2} \sigma^{m_{2}}=\beta_{1} \sigma^{m_{1}} \beta_{2} \sigma^{-m_{1}} \sigma^{m_{1}+m_{2}}=\left(\beta_{1} m_{1} \beta_{2}, m_{1}+m_{2}\right) .
$$

Notice that the decomposition of $\operatorname{Aff}(\mathbb{T})$ depends on the choice of the element $\sigma$.

We say that a subgroup $\Gamma$ of $\operatorname{Aff}(\mathbb{T})$ is exceptional if $\Gamma \subseteq \operatorname{Hor}(\mathbb{T})$ or if $\Gamma$ fixes an element of $\partial^{*} \mathbb{T}$. In this paper we will always consider closed and non-exceptional subgroups $\Gamma$. It is known that $\Gamma$ is non-exceptional if and only if it is non-unimodular. In this case the limit set $\partial \Gamma$ of $\Gamma$, i.e. the set of accumulation points of an orbit $\Gamma o$ in $\partial \mathbb{T}$, is uncountable and $\omega \in \partial \Gamma$. Moreover for $\mathfrak{u} \in \partial \Gamma \backslash\{\omega\}$ the orbit $\Gamma \mathfrak{u}$ is dense in $\partial \Gamma$ (see [4]).

\subsection{Length Functions}

Notice that there exists an unique $\mathfrak{f}=\mathfrak{f}^{\sigma} \in \partial^{*} \mathbb{T}$ such that $\sigma\left(\mathfrak{f}^{\sigma}\right)=\mathfrak{f}^{\sigma}$. Indeed, $\mathfrak{f}$ is represented by the geodesic ray $o, \sigma o, \sigma^{2} o, \ldots$ Then $\sigma$ acts by the translation on $\overline{\mathfrak{f} \omega}$. We define length functions on the boundary $\partial^{*} \mathbb{T}$ and on the affine group:

$$
\begin{aligned}
|\mathfrak{u}| & =\Theta(\mathfrak{u}, \mathfrak{f}), \quad \mathfrak{u} \in \partial^{*} \mathbb{T}, \\
\|\gamma\| & =\Theta(\gamma \mathfrak{f}, \mathfrak{f}), \quad \gamma \in G .
\end{aligned}
$$

Observe that the group $\mathbb{Z}$ is included in the kernel of $\|\cdot\|$ and for any $\gamma=(\beta, m) \in G$ we have $\|\gamma\|=\|\beta\|=\left\|\beta^{-1}\right\|$.

We decompose both the boundary and the affine group with respect to the value of the corresponding length function. For $j \in \mathbb{Z}$ we define

$$
\begin{aligned}
& A_{j}=\left\{\mathfrak{u} \in \partial^{*} \mathbb{T}:|\mathfrak{u}|=q^{j}\right\}=\left\{\mathfrak{u} \in \partial^{*} \mathbb{T}: \mathfrak{u} \wedge \mathfrak{f}=\sigma^{-j} o\right\}, \\
& G_{j}=\left\{g \in G:\|g\|=q^{j}\right\}=\left\{g \in G: g \mathfrak{f} \in A_{j}\right\} .
\end{aligned}
$$

Then $\partial^{*} \mathbb{T}=\{\mathfrak{f}\} \cup \bigcup_{j \in \mathbb{Z}} A_{j}$, and $G=\{g \in G: g \mathfrak{f}=\mathfrak{f}\} \cup \bigcup_{j \in \mathbb{Z}} G_{j}$. It is a worthwhile observing that the stabiliser $\{g \in \operatorname{Hor}(\mathbb{T}): g \mathfrak{f}=\mathfrak{f}\}$ plays the same role as the group of rotations in the real case. However, contrary to the case where the stabiliser of a point is just the similarity group of euclidean space, the stabiliser of the point in the 
boundary of the tree is much more complex since its elements do not commute with the reference homothety $\sigma$.

We will use later some properties of the sets defined above, which are formulated in the following lemma.

Lemma 2.1 Suppose that $(\beta, m) \in G_{j}$ for some $j \in \mathbb{Z}$, then

(i) Both $\beta$ and $\beta^{-1}$ preserve $A_{k}$ and $\sigma^{-k}$ ofor every $k>j$.

(ii) If $\mathfrak{u} \in A_{j}$, then $\beta \mathfrak{u} \in A_{k}$ for some $k \leq j$.

(iii) If $\mathfrak{u} \in A_{k}$ for some $k<j$, then $\beta \mathfrak{u} \in A_{j}$.

Proof Follows easily from

$$
|\mathfrak{u}|=\Theta(\mathfrak{f}, \mathfrak{u})=\Theta(\beta \mathfrak{f}, \beta \mathfrak{u}) \leq \max \{\Theta(\beta \mathfrak{f}, \mathfrak{f}), \Theta(\beta \mathfrak{u}, \mathfrak{f})\}=\max \{\|\beta\|,|\beta \mathfrak{u}|\}
$$

and

$$
|\beta \mathfrak{u}| \leq \max \{\|\beta\|,|\mathfrak{u}|\} .
$$

\subsection{Random Walks on Aff( $\mathbb{T})$ and the Main Theorem}

Let $\mu$ be a probability measure on Aff( $\mathbb{T})$. We will assume that the closed semigroup $\Gamma$ generated by the support of $\mu$ is non-exceptional. For sake of simplicity we will also assume that $\phi(\Gamma)=\mathbb{Z}$.

We define the left and the right random walk on $G$ by $L_{n}=X_{n} X_{n-1} \cdot \ldots \cdot X_{1}$, $R_{n}=X_{1} X_{2} \cdot \ldots \cdot X_{n}$, and $L_{0}=R_{0}=e(e$ is the identity in $G)$. Notice that both processes have different trajectories, but they have the same law, i.e. $L_{n}={ }_{d} R_{n}$. Since $\Gamma$ is non-exceptional it is non-unimodular, hence the random walks $L_{n}$ and $R_{n}$ are transient. By $\bar{\mu}$ we denote the image of the measure $\mu$ on $\mathbb{Z}$, i.e. $\bar{\mu}(k)=\mu\left(\phi^{-1}\{k\}\right)$. Then $\phi\left(L_{n}\right)=\phi\left(R_{n}\right)=\phi\left(X_{1}\right)+\cdots+\phi\left(X_{n}\right)$ is a sum of i.i.d. random variables with law $\bar{\mu}$. If the measure $\bar{\mu}$ has the first moment then by $m_{1}$ we denote its mean $m_{1}=\sum_{k \in \mathbb{Z}} k \bar{\mu}(k)$. The value $m_{1}$ is called drift of $\mu$ and it describes behaviour of the random processes both on the tree and its boundary generated by the action of the group. It was proved in [1,4] that if $m_{1}<0$, then $R_{n} \cdot o$ converges a.s. to $\omega, L_{n} v$ converges to $\omega$ for every $v \in \partial^{*} \mathbb{T}$ and the Markov chain $\left\{L_{n} v\right\}$ is transient. If $m_{1}>0$ and $\mathbb{E}\left[\left|X_{1}\right|\right]<\infty$ then $R_{n} \cdot o$ converges a.s. to some random variable $\xi_{\infty}$ defined on $\partial^{*} \mathbb{T}$. Then the law $\eta$ of $\xi_{\infty}$ is a unique stationary measure of the random process $\left\{L_{n} \xi_{\infty}\right\}$ on $\partial^{*} \mathbb{T}$, which is positive recurrent (see Brofferio [1]).

The most interesting is the drift-free case, when $m_{1}=0$. Then, if $\mathbb{E}\left[\left|X_{1}\right|\right]<\infty$, $R_{n} \cdot o$ converges a.s. to $\omega$. In this situation Brofferio [1] proved that if $\mathbb{E}\left[\phi\left(X_{1}\right)^{2}+\right.$ $\left.\left|\beta\left(X_{1}\right)\right|^{2+\varepsilon}\right]<\infty$, then the chain $\left\{L_{n} v\right\}$ is recurrent and there exists a unique (up to a multiplicative constant) $\mu$-invariant Radon measure $v$ on $\partial^{*} \mathbb{T}$, i.e. the measure satisfying (1.1). The measure $v$ is crucial to obtain the renewal theorem on the affine group. The main purpose of this paper is to describe behaviour of the measure $v$ at infinity. Our main result is the following

Theorem 2.2 Let $\mu$ be a probability measure on the affine group $G$. Assume

$$
\int_{G} \phi(g) \mu(d g)=0,
$$


$\int_{G}\left(q^{\delta m}+q^{-\delta m}+\|\beta\|^{\delta}\right) \mu(d \beta, d m)<\infty \quad$ for some $\delta>0$,

the subgroup generated by the support of $\mu$ is non-exceptional,

the subgroup generated by the support of $\bar{\mu}=\phi(\mu)$ is $\mathbb{Z}$.

Then

$$
\lim _{k \rightarrow+\infty} v\left\{\mathfrak{u}:|\mathfrak{u}|=q^{k}\right\}=C_{+},
$$

for some strictly positive constant $C_{+}$.

\section{Proof of Theorem 2.2}

\subsection{First Properties of the Measure $v$}

To simplify our notation we define a function $v: \mathbb{Z} \mapsto \mathbb{R}$ by the formula $v(k)=$ $v\left(A_{k}\right)$. We are going to prove that $\lim _{k \rightarrow+\infty} v(k)=C_{+}>0$. First we will justify that without any loss of generality, we may assume additionally that for any $1<d<q$ :

$$
\sum_{k \in \mathbb{Z}} d^{-k} v(k)<\infty
$$

We begin with the following lemma.

Lemma 3.1 There exists $n \in \mathbb{Z}$ such that $v(i)>0$ for every $i \geq n$. Moreover there exists $\varepsilon>0$ such that

$$
v(k) \geq \varepsilon^{|k-l|} v(l)
$$

for $k, l \geq n$.

Proof In view of (2.1) and (2.4) there exist $k^{+}, k^{-} \in \mathbb{N}, n_{0} \in \mathbb{Z}$ and $\varepsilon>0$ such that

$$
\mu^{* k^{ \pm}}\left\{(\beta, \pm 1):\|\beta\|<q^{-n_{0}}\right\} \geq \varepsilon
$$

By Lemma 2.1(i) if $\|\beta\|<q^{-n_{0}}$ then $\beta^{-1}$ preserves sets $A_{i \pm 1}$ for every $i>n_{0}$. Hence for $i>n_{0}$

$$
\begin{aligned}
v(i \mp 1) & =v\left(A_{i \mp 1}\right)=\mu^{* k^{ \pm}} * v\left(A_{i \mp 1}\right) \\
& \geq \int_{\left\{(\beta, \pm 1):\|\beta\|<q^{-n_{0}}\right\}} \int_{\partial^{* \mathbb{T}}} \mathbf{1}_{A_{i \mp 1}}(\beta a \mathfrak{u}) v(d \mathfrak{u}) \mu^{* k^{ \pm}}(d \beta, d a) \\
& \geq \mu^{* k \pm}\left(\left\{(\beta, \pm 1):\|\beta\|<q^{-n_{0}}\right\}\right) \int_{\partial^{*} \mathbb{T}} \mathbf{1}_{A_{i}}(\mathfrak{u}) v(d \mathfrak{u}) \\
& \geq \varepsilon v(i) .
\end{aligned}
$$


Since $v$ is an unbounded Radon measure, we can find $n>n_{0}$ such that $v(n)>0$. Therefore in view of (3.2) we have $v(i)>0$ for $i \geq n$. Moreover, for $k, l \geq n$ we obtain

$$
\frac{v(k)}{v(l)} \leq \varepsilon^{-|k-l|},
$$

which finishes the proof.

Proposition 3.2 Suppose that the measure $\mu$ satisfies (2.1)-(2.4). Then

$$
\lim _{i \rightarrow+\infty} \frac{v(i)}{v(i+1)}=1 .
$$

In particular for any $\gamma>1$

$$
\sum_{k=0}^{\infty} v(k) \gamma^{-k}<\infty
$$

Proof Take $n$ as in the previous lemma and fix for a moment $m \in \mathbb{Z}$. Then by the lemma the sequence $\frac{v(m+i)}{v(n+i)}$ is bounded for positive $i$. Hence we can find a sequence $\left\{i_{k}\right\}$ tending to $+\infty$ and a real number $C(m)$ such that

$$
\lim _{k \rightarrow+\infty} \frac{v\left(m+i_{k}\right)}{v\left(n+i_{k}\right)}=C(m) .
$$

Using the diagonal method we can find a sub-sequence $\left\{i_{k_{p}}\right\}$ such that

$$
\lim _{p \rightarrow+\infty} \frac{v\left(m+i_{k_{p}}\right)}{v\left(n+i_{k_{p}}\right)}=C(m)
$$

for every $m \in \mathbb{Z}$. We will prove that the function $C$ on $\mathbb{Z}$, defined above, is $\bar{\mu}$ harmonic, hence constant.

Notice that if we take $(\beta, a) \in G, l \in \mathbb{Z}$ and $i_{k_{p}} \geq \log _{q}\|\beta\|-l$ then by Lemma 2.1(i) both $\beta$ and $\beta^{-1}$ preserve $A_{l+i_{k}}$ hence

$$
\begin{aligned}
\delta_{i_{k_{p}}} * \delta(\beta, a) * v\left(\mathbf{1}_{A_{l}}\right) & =\int_{\partial * \mathbb{T}} \mathbf{1}_{A_{l}}\left(i_{k_{p}} \beta a \mathfrak{u}\right) v(d \mathfrak{u})=\int_{\partial^{*} \mathbb{T}} \mathbf{1}_{A_{l+i_{k}}}(\beta a \mathfrak{u}) v(d \mathfrak{u}) \\
& =\int_{\partial^{*} \mathbb{T}} \mathbf{1}_{A_{l+i_{k} p}}(a \mathfrak{u}) v(d \mathfrak{u})=\int_{\partial^{*} \mathbb{T}} \mathbf{1}_{A_{l}}\left(\left(i_{k_{p}}+a\right) \mathfrak{u}\right) v(d \mathfrak{u}) \\
& =\delta_{i_{k_{p}}} * \delta_{(0, a)} * v\left(\mathbf{1}_{A_{l}}\right),
\end{aligned}
$$

where 0 denotes the identity element in the group Hor $(\mathbb{T})$. Therefore, by the Fatou lemma and the invariance of $v$, we have

$$
\begin{aligned}
\int_{\mathbb{Z}} C(l+a) \bar{\mu}(d a) & =\int_{\mathbb{Z}} \lim _{p \rightarrow+\infty} \frac{1}{v\left(n+i_{k_{p}}\right)} \delta_{i_{k_{p}}} * \delta_{(0, a)} * v\left(\mathbf{1}_{A_{l}}\right) \bar{\mu}(d a) \\
& =\int_{G} \lim _{p \rightarrow+\infty} \frac{1}{v\left(n+i_{k_{p}}\right)} \delta_{i_{k_{p}}} * \delta_{(\beta, a)} * v\left(\mathbf{1}_{A_{l}}\right) \mu(d \beta, d a)
\end{aligned}
$$




$$
\begin{aligned}
& \leq \liminf _{p \rightarrow+\infty} \frac{1}{v\left(n+i_{k_{p}}\right)} \int_{G} \delta_{i_{k_{p}}} * \delta(\beta, a) * v\left(\mathbf{1}_{A_{l}}\right) \mu(d \beta, d a) \\
& =\liminf _{p \rightarrow+\infty} \frac{v\left(l+i_{k_{p}}\right)}{v\left(n+i_{k_{p}}\right)}=C(l) .
\end{aligned}
$$

Since the measure $\bar{\mu}$ is recurrent and $C$ is $\bar{\mu}$-superharmonic, $C$ is $\bar{\mu}$-harmonic, hence constant. But $C(n)=1$, so it follows that $C \equiv 1$. Summarising, we have proved that for any subsequence $\left\{i_{k}\right\}$ of integers there exists its subsequence $\left\{i_{k_{l}}\right\}$ such that

$$
\lim _{l \rightarrow+\infty} \frac{v\left(m+i_{k_{l}}\right)}{v\left(n+i_{k_{l}}\right)}=1
$$

for every $m \in \mathbb{Z}$. Therefore

$$
\lim _{i \rightarrow+\infty} \frac{v(m+i)}{v(n+i)}=1
$$

and taking $m=n-1$ we obtain (3.3). The second statement follows now easily from the ratio criterion.

Lemma 3.3 For any $1<d<q$ there exists $\beta_{0} \in \operatorname{Hor}(\mathbb{T})$ such that the measure $\tilde{v}=$ $\delta_{\beta_{0}} * v$ satisfies:

$$
\sum_{k \in \mathbb{Z}} d^{-k} \tilde{v}(k)<\infty
$$

Proof Let us observe that the translated measure $\tilde{v}=\delta_{\beta} * v$ has the same behaviour at infinity as the measure $\nu$. Indeed, by Lemma 2.1(i) for $k>\log \|\beta\|$,

$$
\tilde{v}\left(A_{k}\right)=v\left(A_{k}\right) .
$$

In view of Proposition 3.2 it is enough to consider only the sum over negative $k$ 's.

Let $m_{r}$ be the right Haar measure on $G$. By Soardi and Woess [7], $G$ is nonunimodular with the modular function $g \mapsto q^{\phi(g)}$ for $g \in G$, hence $m_{r}\left(g A g^{-1}\right)=$ $q^{-\phi(g)} m_{r}(A)$ for any Borel set $A$. By $H$ let us denote the stabilizer of $o$ in $G$. If we write $H_{-\infty}=\{\beta \in \operatorname{Hor}(\mathbb{T}): \beta \mathfrak{f}=\mathfrak{f}\}$ and $H_{k}=G_{k} \cap \operatorname{Hor}(\mathbb{T})$ for $k \in \mathbb{Z}$, then $H=H_{-\infty} \cup \bigcup_{k \leq 0} H_{k}$.

Since $H$ is open and compact its Haar measure is strictly positive and finite. Moreover from

$$
m_{r}\left(H_{k}\right)=m_{r}\left(\sigma^{-k} H_{0} \sigma^{k}\right)=q^{-k} \cdot m_{r}\left(H_{0}\right)
$$

and

$$
m_{r}\left(H_{-\infty}\right)=m_{r}\left(\bigcap_{k \leq 0} \sigma^{-k} H \sigma^{k}\right)=\lim _{k \rightarrow-\infty} q^{k} m_{r}(H)=0
$$

it follows that $m_{r}(H)=\frac{q}{q-1} m_{r}\left(H_{0}\right)$. 
Fix $0<\gamma<1$. For every $\mathfrak{u} \in U=\{|\mathfrak{u}|<1\}$ there exists $\beta_{\mathfrak{u}} \in H$ satisfying $\beta_{\mathfrak{u}} \mathfrak{f}=$ $\mathfrak{u}$. Since $H \beta_{\mathfrak{u}}=H,|\beta \mathfrak{f}|^{-\gamma}$ is positive and $m_{r}$-a.e. finite, we have

$$
\int_{H} \frac{1}{|\beta \mathfrak{u}|^{\gamma}} m_{r}(d \beta)=\int_{H} \frac{1}{\left|\beta \beta_{\mathfrak{u}}\right|^{\gamma}} m_{r}(d \beta)=\int_{H \beta_{\mathfrak{u}}} \frac{1}{|\beta \mathfrak{f}|^{\gamma}} m_{r}(d \beta)=\int_{H} \frac{1}{|\beta \mathfrak{f}|^{\gamma}} m_{r}(d \beta) .
$$

Now we can write

$$
\begin{aligned}
& \int_{H} \int_{U} \frac{1}{|\beta \mathfrak{u}|^{\gamma}} v(d \mathfrak{u}) m_{r}(d \beta) \\
& \quad=\int_{U} \int_{H} \frac{1}{|\beta \mathfrak{u}|^{\gamma}} m_{r}(d \beta) v(d \mathfrak{u})=v(U) \int_{H} \frac{1}{|\beta \mathfrak{f}|^{\gamma}} m_{r}(d \beta) \\
& =v(U) \sum_{k \leq 0} \int_{H_{k}} \frac{1}{|\beta \mathfrak{f}|^{\gamma}} m_{r}(d \beta)=v(U) \sum_{k \leq 0} \int_{H_{k}} q^{-k \gamma} m_{r}(d \beta) \\
& =v(U) m\left(H_{0}\right) \sum_{k \leq 0} q^{k-k \gamma}<\infty .
\end{aligned}
$$

Take arbitrary $\beta_{0} \in H, 1<d<q$ and denote $\gamma=\log _{q} d$. If $\beta_{0} \mathfrak{u} \in A_{k}$, then $d^{k}=\left|\beta_{0} \mathfrak{u}\right|^{\gamma}$. Thus

$$
\begin{aligned}
\sum_{k \leq 0} d^{-k} \cdot \delta_{\beta_{0}} * v\left(A_{k}\right) & =\sum_{k \leq 0} \int_{\partial^{*} \mathbb{T}} d^{-k} \mathbf{1}_{A_{k}}\left(\beta_{0} \mathfrak{u}\right) v(d \mathfrak{u}) \\
& =\int_{\partial^{*} \mathbb{T}}\left[\frac{1}{\left|\beta_{0} \mathfrak{u}\right|^{\gamma}} \sum_{k \leq 0} \mathbf{1}_{A_{k}}\left(\beta_{0} \mathfrak{u}\right)\right] v(d \mathfrak{u})=\int_{U} \frac{1}{\left|\beta_{0} \mathfrak{u}\right|^{\gamma}} v(d \mathfrak{u})
\end{aligned}
$$

and by (3.6) there exists $\beta_{0} \in H_{0}$ such that the value above is finite.

Take $\beta_{0}$ as in the lemma. Then the translated measure $\tilde{v}=\delta_{\beta_{0}} * v$ has the same behaviour at infinity as the measure $v$. But the measure $\tilde{v}$ is the unique invariant measure of $\tilde{\mu}=\delta_{\beta_{0}} * \mu * \delta_{\beta_{0}^{-1}}$, and obviously $\tilde{\mu}$ satisfies conditions (2.1)-(2.4). Hence to prove Theorem 2.2 it is enough to consider measures $\tilde{v}$ and $\tilde{\mu}$ instead of $v$ and $\mu$. However to simplify our notation we will just use symbols $v, \mu$ and assume that (3.1) is satisfied.

\subsection{The Poisson Equation}

In order to prove Theorem 2.2 we will consider $v$ as a solution of the Poisson equation

$$
\bar{\mu} * v(k)=v(k)+\psi(k)
$$

for $\psi$ defined by the equation above, i.e. $\psi=\bar{\mu} * v-v$. It was proved by Spitzer [8] that if the function $\psi$ is sufficiently good, there exists an explicit formula describing all nonnegative solutions of the Poisson equation. 
Lemma 3.4 Suppose that hypotheses (2.1)-(2.4) and condition (3.1) are fulfilled. Then the function $\psi=\bar{\mu} * v-v$ satisfies

$$
\sum_{k \in \mathbb{Z}}|k \psi(k)|<\infty
$$

and

$$
\sum_{k \in \mathbb{Z}} \psi(k)=0
$$

Proof In view of Lemma 2.1, the function $\psi$ can be written as follows

$$
\begin{aligned}
\psi(k) & =\bar{\mu} * v\left(\mathbf{1}_{A_{k}}\right)-v\left(\mathbf{1}_{A_{k}}\right)=\bar{\mu} * v\left(\mathbf{1}_{A_{k}}\right)-\mu * v\left(\mathbf{1}_{A_{k}}\right) \\
& =\int_{G} \int_{\partial^{* T}}\left(\mathbf{1}_{A_{k}}(m \mathfrak{u})-\mathbf{1}_{A_{k}}(\beta m \mathfrak{u})\right) v(d \mathfrak{u}) \mu(d \beta, d m) \\
& =\sum_{j \geq k} \int_{G_{j}} \int_{\partial * \mathbb{T}}\left(\mathbf{1}_{A_{k}}(m \mathfrak{u})-\mathbf{1}_{A_{k}}(\beta m \mathfrak{u})\right) v(d \mathfrak{u}) \mu(d \beta, d m) .
\end{aligned}
$$

Next we write $\psi \leq \psi_{1}+\psi_{2}$, where

$$
\begin{aligned}
& \psi_{1}(k)=\sum_{j>k} \int_{G_{j}} \int_{\partial^{*} \mathbb{T}}\left|\mathbf{1}_{A_{k}}(\beta m \mathfrak{u})-\mathbf{1}_{A_{k}}(m \mathfrak{u})\right| v(d \mathfrak{u}) \mu(d \beta, d m), \\
& \psi_{2}(k)=\int_{G_{k}} \int_{\partial^{*} \mathbb{T}}\left|\mathbf{1}_{A_{k}}(\beta m \mathfrak{u})-\mathbf{1}_{A_{k}}(m \mathfrak{u})\right| v(d \mathfrak{u}) \mu(d \beta, d m) .
\end{aligned}
$$

We will show that both $k \psi_{1}(k)$ and $k \psi_{2}(k)$ are summable. First we will prove that

$$
\sum_{k \in \mathbb{Z}}|k| \psi_{2}(k)<\infty
$$

Notice that if $\beta \in G_{k}$, then by Lemma 2.1 we have

$$
\left|\mathbf{1}_{A_{k}}(\beta \mathfrak{u})-\mathbf{1}_{A_{k}}(\mathfrak{u})\right| \leq \mathbf{1}_{\bigcup_{j \leq k} A_{j}}(\mathfrak{u}) .
$$

Take $\delta$ as in (2.2), fix $\delta^{\prime}<\frac{\delta}{2}$ and let $d=q^{\delta^{\prime}}$. Then we have

$$
\begin{aligned}
\sum_{k \in \mathbb{Z}}|k| \psi_{2}(k) & \leq \sum_{k}|k| \int_{G_{k}} \int_{\partial^{*} \mathbb{T}} \mathbf{1}_{\bigcup_{j \leq k} A_{j}}(m \mathfrak{u}) v(d \mathfrak{u}) \mu(d \beta, d m) \\
& =\sum_{k \in \mathbb{Z}}|k| \int_{G_{k}} \int_{\partial^{*} \mathbb{T}} \mathbf{1}_{\bigcup_{j \leq k} A_{j+m}}(\mathfrak{u}) v(d \mathfrak{u}) \mu(d \beta, d m) \\
& =\sum_{k \in \mathbb{Z}}|k| \int_{G_{k}} \sum_{j \leq k} v(j+m) \mu(d \beta, d m)
\end{aligned}
$$




$$
\begin{aligned}
& =\sum_{k \in \mathbb{Z}} \int_{G_{k}} d^{m}|k| \sum_{j \leq k} d^{j} d^{-(j+m)} v(j+m) \mu(d \beta, d m) \\
& \leq \sum_{k \in \mathbb{Z}} \int_{G_{k}} d^{m}|k| d^{k}\left(\sum_{j \in \mathbb{Z}} d^{-(j+m)} v(j+m)\right) \mu(d \beta, d m) \\
& \leq\left(\sum_{j \in \mathbb{Z}} d^{-j} v(j)\right) \sum_{k \in \mathbb{Z}} \int_{G_{k}} d^{m}|k| d^{k} \mu(d \beta, d m) .
\end{aligned}
$$

In view of (3.1) the first expression is finite. To prove finiteness of the second one recall that if $\beta \in G_{k}$, then $k=\log _{q}\|\beta\|$ and write

$$
\begin{aligned}
& \sum_{k \in \mathbb{Z}} \int_{G_{k}} d^{m}|k| d^{k} \mu(d \beta, d m) \\
& \quad=\int_{G} q^{\delta^{\prime} m}\left|\log _{q}\|\beta\|\right|\|\beta\|^{\delta^{\prime}} \mu(d \beta, d m) \\
& \quad \leq\left(\int_{G} q^{2 \delta^{\prime} m} \mu(d \beta, d m)\right)^{\frac{1}{2}} \cdot\left(\int_{G}\left(\log _{q}^{2}\|\beta\|\right)\|\beta\|^{2 \delta^{\prime}} \mu(d \beta, d m)\right)^{\frac{1}{2}} .
\end{aligned}
$$

By (2.2) the expression above is bounded and we obtain (3.10).

Now we are going to prove that

$$
\sum_{k \in \mathbb{Z}}|k| \psi_{1}(k)<\infty
$$

Notice first that

$$
\psi_{1}(k) \leq \psi_{1}^{1}(k)+\psi_{1}^{2}(k)
$$

where

$$
\begin{aligned}
& \psi_{1}^{1}(k)=\sum_{j>k} \int_{G_{j}} \int_{\partial^{*} \mathbb{T}} \mathbf{1}_{A_{k}}(\beta m \mathfrak{u}) v(d \mathfrak{u}) \mu(d \beta, d m), \\
& \psi_{1}^{2}(k)=\sum_{j>k} \int_{G_{j}} v(k+m) \mu(d \beta, d m) .
\end{aligned}
$$

To prove that $\sum_{k \in \mathbb{Z}}|k| \psi_{1}^{2}<\infty$ we use exactly the same estimates as above. To estimate $\psi_{1}^{1}$ we deal first with negative $k$. Then by (3.1) we have

$$
\begin{aligned}
\sum_{k<0}|k| \psi_{1}^{1}(k) & =\sum_{k<0}|k| \sum_{j>k} \int_{G_{j}} \int_{\partial^{*} \mathbb{T}} \mathbf{1}_{A_{k}}(\beta m \mathfrak{u}) v(d \mathfrak{u}) \mu(d \beta, d m) \\
& \leq \sum_{k<0}|k| \cdot \mu * v\left(A_{k}\right)=\sum_{k<0}|k| v(k)<\infty .
\end{aligned}
$$


Finally, for $\delta^{\prime \prime} \leq \frac{\delta}{2}, \delta^{\prime}<\delta^{\prime \prime}, d=q^{\delta^{\prime \prime}}$

$$
\begin{aligned}
\sum_{k>0} k \psi_{1}^{1}(k) & =\sum_{k>0} k \sum_{j>k} \int_{G_{j}} \int_{\partial^{*} \mathbb{T}} \mathbf{1}_{A_{k}}(\beta m \mathfrak{u}) v(d \mathfrak{u}) \mu(d \beta, d m) \\
& \leq \sum_{j>0} \int_{G_{j}} \sum_{0<k<j} j \int_{\partial^{*} \mathbb{T}} \mathbf{1}_{A_{k}}(\beta m \mathfrak{u}) v(d \mathfrak{u}) \mu(d \beta, d m) \\
& \leq \sum_{j>0} \int_{G_{j}} j^{2} \int_{\partial^{*} \mathbb{T}} \mathbf{1}_{A_{j}}(m \mathfrak{u}) v(d \mathfrak{u}) \mu(d \beta, d m) \\
& =\sum_{j>0} \int_{G_{j}} j^{2} v(j+m) \mu(d \beta, d m) \\
& \leq \int_{G}\|\beta\|^{\delta^{\prime \prime}} \sum_{j>0}\left(d^{-j} j^{2} v(j+m)\right) \mu(d \beta, d m) \\
& \leq C \int_{G}\|\beta\|^{\delta^{\prime \prime}} q^{\delta^{\prime} m}\left(\sum_{j \in \mathbb{Z}} q^{-\delta^{\prime}(j+m)} v(j+m)\right) \mu(d \beta, d m) \\
& \leq C\left(\sum_{j \in \mathbb{Z}} q^{-\delta^{\prime} j} v(j)\right) \int_{G}\|\beta\|^{\delta^{\prime \prime}} q^{\delta^{\prime} m} \mu(d \beta, d m) .
\end{aligned}
$$

By (3.1) and (2.2) the expression above is finite and we obtain (3.11). The arguments used above give also

$$
\sum_{k \in \mathbb{Z}} \int_{G} \int_{\partial^{*} \mathbb{T}}\left|\mathbf{1}_{A_{k}}(\beta m \mathfrak{u})-\mathbf{1}_{A_{k}}(m \mathfrak{u})\right| v(d \mathfrak{u}) \mu(d \beta, d m)<\infty
$$

therefore by the Fubini theorem

$$
\begin{aligned}
& \sum_{k} \int_{G} \int_{\partial^{*} \mathbb{T}}\left(\mathbf{1}_{A_{k}}(\beta m \mathfrak{u})-\mathbf{1}_{A_{k}}(m \mathfrak{u})\right) v(d \mathfrak{u}) \mu(d \beta, d m) \\
& \quad=\int_{G} \int_{\partial^{*} \mathbb{T}} \sum_{k \in Z}\left(\mathbf{1}_{A_{k}}(\beta m \mathfrak{u})-\mathbf{1}_{A_{k}}(m \mathfrak{u})\right) v(d \mathfrak{u}) \mu(d \beta, d m)=0 .
\end{aligned}
$$

\subsection{Proof of Theorem 2.2-Existence of the Limit}

The result follows from a theorem of Spitzer [8, p. 375], who proved that if $\sigma^{2}=$ $\sum_{k \in \mathbb{Z}} k^{2} \bar{\mu}(d k)<\infty$ and $\sum_{k \in \mathbb{Z}}|k \psi(k)|<\infty$, then all positive solutions of the Poisson equation (3.7) are of the form

$$
v(k)=\psi * a(k)+c_{2} k+c_{3} .
$$


In the formula above $a$ is the recurrent potential defined by the formula

$$
a(k)=\lim _{n \rightarrow \infty} \sum_{i=1}^{n}\left(\bar{\mu}^{* i}(0)-\bar{\mu}^{* i}(k)\right)
$$

and $c_{2}$ is some constant satisfying

$$
\sigma^{2}\left|c_{2}\right| \leq \sum_{k \in \mathbb{Z}} \psi(k) .
$$

In fact, the theorem was proved for finitely supported functions $\psi$, nevertheless the proof is valid also under weaker assumptions i.e. for functions satisfying $\sum_{k \in \mathbb{Z}}|k \psi(k)|<\infty$ (see the second paragraph on p. 376 in [8] and also the Appendix in [3]).

Since $\sum_{k \in \mathbb{Z}} \psi(k)=0$, the constant $c_{2}$ must be zero. We will need the following property of $a$ :

$$
\lim _{k \rightarrow \pm \infty}(a(k+n)-a(k))=\mp \frac{n}{\sigma^{2}} .
$$

By (3.1), $\lim _{k \rightarrow-\infty} v(k)=0$, therefore

$$
\begin{aligned}
0 & =\lim _{k \rightarrow-\infty} v(k)=\lim _{k \rightarrow-\infty} \sum_{n \in \mathbb{Z}} \psi(n) a(k-n)+c_{3} \\
& =\lim _{k \rightarrow-\infty} \sum_{n \in \mathbb{Z}} \psi(n)(a(k-n)-a(k))+c_{3}=-\frac{1}{\sigma^{2}} \sum_{n \in \mathbb{Z}} n \psi(n)+c_{3} .
\end{aligned}
$$

So we obtain $c_{3}=\frac{1}{\sigma^{2}} \sum_{n \in \mathbb{Z}} n \psi(n)$. Finally we compute

$$
\begin{aligned}
\lim _{k \rightarrow+\infty} v(k) & =\lim _{k \rightarrow+\infty} \sum_{n \in \mathbb{Z}} \psi(n) a(k-n)+c_{3} \\
& =\lim _{k \rightarrow+\infty} \sum_{n \in \mathbb{Z}} \psi(n)(a(k-n)-a(k))+c_{3}=\frac{2}{\sigma^{2}} \sum_{n \in \mathbb{Z}} n \psi(n)=C_{+} .
\end{aligned}
$$

\subsection{Proof of Theorem 2.2-Positivity of the Limiting Constant}

Now we are going to prove that the constant $C_{+}$is strictly positive. We will apply to our settings arguments given in [2] for the real affine group. Notice that it is enough to prove that there exists $C>0$ and $M$ such that for any positive nonincreasing and bounded sequence $\left\{a_{k}\right\}_{k \in \mathbb{Z}}$

$$
\sum_{k \in \mathbb{Z}} a_{k} v(k) \geq C \sum_{k=M}^{\infty} a_{k} .
$$

Indeed, assume that $\lim _{k \rightarrow+\infty} v(k)=0$. Then for any $\varepsilon>0$ there exists $N$ such that $v(k)<\varepsilon$ for $k>N$. Let us substitute $a_{k}=1$ for $k \leq n$ and $a_{k}=0$ for $k>n$. Since $v$ 
is the Radon measure, $\sum_{k=-\infty}^{N-1} v(k)=v\left\{\mathfrak{u} \in \partial^{*} \mathbb{T}:|\mathfrak{u}| \leq q^{N-1}\right\}<\infty$, therefore

$$
\lim _{n \rightarrow+\infty} \frac{1}{n} \sum_{k \in \mathbb{Z}} a_{k} v(k)=\lim _{n \rightarrow+\infty} \frac{1}{n} \sum_{k=N}^{n} v(k)+\lim _{n \rightarrow+\infty} \frac{1}{n} \sum_{k=-\infty}^{N-1} v(k) \leq \varepsilon,
$$

but

$$
\lim _{n \rightarrow+\infty} \frac{C}{n} \sum_{k=M}^{\infty} a_{k}=C .
$$

Therefore, in view of (3.12), $0<C \leq \varepsilon$, but this inequality cannot be true for arbitrary small $\varepsilon$. So we deduce $\limsup _{k \rightarrow \infty} v(k)>0$.

In order to prove (3.12) we will use an explicit construction of the measure $v$. Let us define strictly ascending ladder epochs i.e. a sequence of stopping times $l_{n+1}=$ $\inf \left\{k>l_{n}: S_{k}>S_{l_{n}}\right\}, l_{0}=0$, where $S_{k}=\phi\left(L_{k}\right)$ (see [5, p. 391]). Then $L_{l_{n}}$ is a random walk on $G$ with a positive drift, therefore there exists a probability measure $\eta$ on $\partial^{*} \mathbb{T}$, which is the unique stationary measure of the process $\left\{L_{l_{n}}\right\}$ (see [1] for more details). The measure $v$ can be written (up to a multiplicative constant) as

$$
v(f)=\int_{\partial^{*} \mathbb{T}}\left[\sum_{k=0}^{l_{1}-1} f\left(L_{k} \cdot \mathfrak{u}\right)\right] \eta(d \mathfrak{u}) .
$$

Now take any nonincreasing, positive, bounded sequence $\left\{a_{n}\right\}_{n \in \mathbb{Z}}$ and define a function on the sequence $\left\{q^{n}\right\}_{n \in \mathbb{Z}}: f\left(q^{k}\right)=a_{k}$. Take a ball $B=\left\{\mathfrak{u}:|\mathfrak{u}| \leq q^{M}\right\}$ for some $M$ and such that $\eta(B)=\varepsilon>0$. Then

$$
\begin{aligned}
\sum_{k \in \mathbb{Z}} a_{k} v(k) & =\int_{\partial^{*} \mathbb{T}} f(|\mathfrak{u}|) v(d \mathfrak{u}) \geq \int_{B} \mathbb{E}\left[\sum_{k=0}^{l_{1}-1} f\left(\left|L_{k} \cdot \mathfrak{u}\right|\right)\right] \eta(d \mathfrak{u}) \\
& =\int_{B} \mathbb{E}\left[\sum_{k=0}^{l_{1}-1} f\left(\left|\beta_{k} \sigma^{m_{k}} \beta_{k-1} \sigma^{m_{k-1}} \ldots \beta_{1} \sigma^{m_{1}} \cdot \mathfrak{u}\right|\right)\right] \eta(d \mathfrak{u}) .
\end{aligned}
$$

Notice that for $\mathfrak{u} \in B$

$$
\begin{aligned}
& \left|\beta_{k} \sigma^{m_{k}} \beta_{k-1} \sigma^{m_{k-1}} \ldots \beta_{1} \sigma^{m_{1}} \cdot \mathfrak{u}\right| \\
& \quad=\left|\sigma^{S_{k}} \sigma^{-S_{k}} \beta_{k} \sigma^{S_{k}} \sigma^{-S_{k-1}} \beta_{k-1} \sigma^{S_{k-1}} \ldots \beta_{1} \cdot \mathfrak{u}\right| \\
& \quad \leq q^{-S_{k}} \max \left\{\left\|\sigma^{-S_{k}} \beta_{k} \sigma^{S_{k}}\right\|, \ldots,\left\|\sigma^{-S_{1}} \beta_{1} \sigma^{S_{1}}\right\|, q^{M}\right\} \\
& \quad=q^{-S_{k}} \max \left\{q^{S_{k}}\left\|\beta_{k}\right\|, \ldots, q^{S_{1}}\left\|\beta_{1}\right\|, q^{M}\right\} \\
& \quad \leq q^{-S_{k}} \max \left\{\left\|\beta_{k}\right\|, \ldots,\left\|\beta_{1}\right\|, q^{M}\right\} .
\end{aligned}
$$

Therefore, applying an extended version for time reversible functions (see [2, Lemma 5.3] for more details and precise statement of the duality lemma) of the clas- 
sical duality lemma (see [5, XII.2, p. 394])

$$
\begin{aligned}
\sum_{k \in \mathbb{Z}} a_{k} v(k) & \geq \int_{B} \mathbb{E}\left[\sum_{k=0}^{l_{1}-1} f\left(\max _{\mathfrak{u} \in B}\left|\beta_{k} \sigma^{m_{k}} \beta_{k-1} \sigma^{m_{k-1}} \ldots \beta_{1} \sigma^{m_{1}} \cdot \mathfrak{u}\right|\right)\right] \eta(d \mathfrak{u}) \\
& \geq \varepsilon \mathbb{E}\left[\sum_{k=0}^{l_{1}-1} f\left(q^{-S_{k}} \max \left\{\left\|\beta_{k}\right\|,\left\|\beta_{k-1}\right\|,\left\|\beta_{k-2}\right\|, \ldots,\left\|\beta_{1}\right\|, q^{M}\right\}\right)\right] \\
& =\varepsilon \mathbb{E}\left[\sum_{k=0}^{\infty} f\left(q^{-S_{T_{k}} \max }\left\{\left\|\beta_{1}\right\|, \ldots,\left\|\beta_{T_{k}}\right\|, q^{M}\right\}\right)\right],
\end{aligned}
$$

where $\left\{T_{k}\right\}_{k \in \mathbb{N}}$ is a sequence of stopping times: $T_{0}=0, T_{k}=\inf \left\{n>T_{k-1}: S_{n}<\right.$ $\left.S_{T_{k-1}}\right\}$. Observe that the random variables

$$
W_{k}=-\left(S_{T_{k}}-S_{T_{k-1}}\right)+\max _{T_{k-1}<i \leq T_{k}}\left\{\log _{q}\left\|\beta_{i}\right\|\right\} \vee M
$$

are i.i.d., positive, integrable (cf. Proposition 4 in the Appendix in [4]) and satisfy

$$
q^{-S_{T_{k}} \max }\left\{\left\|\beta_{1}\right\|, \ldots,\left\|\beta_{T_{k}}\right\|, q^{M}\right\} \leq q^{\sum_{i=1}^{k} W_{i}} .
$$

Therefore by the renewal theorem there exist $p$ and $N$ such that

$$
\inf _{i \geq N} \sum_{k \geq 0} \mathbb{P}\left[\sum_{j=1}^{k} W_{j} \in[i, i+p)\right] \geq \delta>0 .
$$

Finally

$$
\begin{aligned}
\sum_{k \in \mathbb{Z}} a_{k} v(k) & \geq \varepsilon \sum_{k=0}^{\infty} \mathbb{E}\left[f\left(q^{\sum_{i=1}^{k} W_{i}}\right)\right] \geq \varepsilon \sum_{k=0}^{\infty} \sum_{j=N}^{\infty} a_{j} \mathbb{P}\left[\sum_{i=1}^{k} W_{i}=j\right] \\
& \geq \varepsilon \sum_{n=1}^{\infty} a_{N+p n} \mathbb{P}\left[\sum_{i=1}^{k} W_{i} \in[N+(n-1) p, N+n p)\right] \\
& \geq \varepsilon \delta \sum_{n=1}^{\infty} a_{N+n p} \geq \frac{\varepsilon \delta}{p} \sum_{j=N+p}^{\infty} a_{j},
\end{aligned}
$$

which proves (3.12).

Open Access This article is distributed under the terms of the Creative Commons Attribution Noncommercial License which permits any noncommercial use, distribution, and reproduction in any medium, provided the original author(s) and source are credited.

\section{References}

1. Brofferio, S.: Renewal theory on the affine group of an oriented tree. J. Theor. Probab. 17(4), 819-859 (2004) 
2. Brofferio, S., Buraczewski, D., Damek, E.: On the invariant measure of the random difference equation $X_{n}=A_{n} X_{n-1}+B_{n}$ in the critical case. Preprint, arXiv:0809.1864

3. Buraczewski, D.: On invariant measures of stochastic recursions in a critical case. Ann. Appl. Probab. 17(4), 1245-1272 (2007)

4. Cartwright, D.I., Kaimanovich, V.A., Woess, W.: Random walks on the affine group of local fields and of homogeneous trees. Ann. Inst. Fourier (Grenoble) 44(4), 1243-1288 (1994)

5. Feller, W.: An Introduction to Probability Theory and Its Application II, 2nd edn. Wiley, New York (1971)

6. Kolesko, K.: Asymptotic properties of harmonic measures on homogeneous trees. Colloq. Math. 118(2), 525-537 (2010)

7. Soardi, P.M., Woess, W.: Amenability, unimodularity, and the spectral radius of random walks on infinite graphs. Math. Z. 205 (1990)

8. Spitzer, F.: Principles of random walk. The University Series in Higher Mathematics. Van Nostrand, Princeton (1964) 